Corresponding author: saquib.lakhani@yale.edu

C) 2020 AbuBakr et al. This article is distributed under the terms of the Creative Commons Attribution-NonCommercial License, which permits reuse and redistribution, except for commercial purposes, provided that the original author and source are credited.

Ontology terms: bicuspid aortic valve; bilateral cryptorchidism; dysplastic pulmonary valve; elbow flexion contracture; excessive wrinkled skin; joint laxity; mild global developmental delay; nasogastric tube feeding in infancy; recurrent infections in infancy and early childhood; short stature; synostosis involving bones of the hand

Published by Cold Spring Harbor Laboratory Press

doi:10.1101/mcs.a005207

\section{A novel variant in MAP3K7 associated with an expanded cardiospondylocarpofacial syndrome phenotype}

\author{
Fatima AbuBakr, ${ }^{1}$ Lauren Jeffries, ${ }^{1}$ Weizhen $\mathrm{Ji}^{1}{ }^{1}$ James M. McGrath, ${ }^{2}$ \\ and Saquib A. Lakhani ${ }^{1}$ \\ ${ }^{1}$ Pediatric Genomics Discovery Program, Department of Pediatrics, ${ }^{2}$ Department of Genetics, Yale University \\ School of Medicine, New Haven, Connecticut 06510, USA
}

[Supplemental material is available for this article.]

\section{PATIENT PRESENTATION}

Patient 1 was born at 41 and 4/7 wk gestation after a pregnancy notable only for emesis during the second trimester. His birth weight was 7 pounds and 12 ounces, and Apgar scores were 7 and 8 . A benign sacral dimple, shortened limbs, and small hands and feet with shortened digits were noticed at birth. A heart murmur and respiratory distress led to identification of pulmonary valve stenosis. He was hospitalized in the NICU for the first 5-6 wk of life primarily for feeding issues.

Now an 18-yr-old male, Patient 1 has had numerous clinical evaluations. For many years his working diagnosis clinically, but not genetically, was Noonan syndrome. Prior negative genetic testing had included a karyotype, microarray, proband-only exome sequencing, fragile $X$ testing, subtelomeric fluorescence in situ hybridization (FISH), FISH for 22q11.2 as well as $X, Y, 13,18$, and 21, and 7-dehydrocholesterol levels. Specific gene sequencing 
of the MID1 and PTPN11 genes had been performed without revealing any variants of concern. Patient 1 has a number of multisystem findings which are detailed below (Table 1).

Gastrointestinal: Feeding issues persisted throughout childhood. He had frequent emesis and was admitted for failure to thrive three times in the first few years of life. He was diagnosed with dysphagia and microaspiration during feeds and oral aversion, and he had a nutritionist and feeding therapist for years. He was diagnosed with Crohn's disease at age $10 \mathrm{yr}$ for which he continues to receive infliximab and methylprednisolone.

Cardiovascular: His pulmonary valve stenosis was managed medically until age 26 mo when he required balloon valvuloplasty; no additional cardiac interventions have been required. His most recent echocardiogram at age $15 \mathrm{yr}$ showed bicuspid aortic valve with mild stenosis and mild regurgitation. The mitral and pulmonary valves were mildly stenotic with mild to moderate right ventricular dilation. He experienced a possible cardiac arrest at induction of anesthesia during a routine tympanostomy surgery at age $15 \mathrm{yr}$ but recovered without significant sequelae.

Musculoskeletal: He was treated with growth hormone for short stature. His most recent height at age $17 \mathrm{yr} 11$ mo was $152.1 \mathrm{~cm}(z=-3.25)$. He was identified in infancy to have congenital cervical vertebral fusions of C2-3 (anterior) and C5-7 (posterior, possibly anterior as well) and minimal pectus, which spontaneously resolved. At age $7 \mathrm{yr}$, he was diagnosed with avascular necrosis of the hip with subluxation of the femoral head. Hand and foot abnormalities became more noticeable with age (Fig. 1C-F). Hand $X$ ray at age $13 \mathrm{yr}$ demonstrated bilateral accessory proximal epiphyses within the second metacarpal and synostosis between the right lunate and triquetrum (Fig. 1I). He has widely spaced first and second toes (Fig. 1F). Incomplete extension of the elbows was noted at age $14 \mathrm{mo}$, and by his teen years he was diagnosed with fusion at the elbow (Fig. 1H). He has mild thoracolumbar scoliosis with minimal L5 on S1 anterolisthesis (Fig. 1J,K) and has had no specific treatment for this.

Infections/Pulmonology/Otolaryngology: Upper and lower respiratory infections have been numerous. He had three bouts of pneumonia from age 6 to 7 . He continues to have frequent ear infections to date with several otolaryngologic procedures including tonsillectomy and adenoidectomy (both requiring revisions) and six tympanostomy tube surgeries. He has conductive hearing loss and has been prescribed hearing aids.

Craniofacial: He has distinctive facial features including a prominent forehead with bitemporal narrowing, downslanted palpebral fissures, upturned nares, and small posteriorly rotated ears (Fig. 1A,B). Prior exam features included high narrow palate with dental crowding requiring orthodontia but without a palate expander.

Ophthalmology: He developed strabismus by age $7 \mathrm{yr}$, which was initially treated with glasses, though he required surgery at age 11 and $16 \mathrm{yr}$.

Integumentary: His skin has been described as soft. He has a wrinkled appearance of the soles and palms though without deep creases (Fig. 1G).

Genitourinary: He had orchiopexy for undescended testicles at age $1 \mathrm{yr}$.

Neurodevelopmental: He had a normal brain MRI, but had mild developmental delay (gross and fine motor) and speech delay. Occupational therapy assisted with feeding difficulties and adapting to life with short stature and digits. He was on stimulant therapy in the past for behavioral issues. He repeated first grade primarily as a result of school absences for frequent illness.

\section{Technical Analysis}

Genomic DNA was prepared from blood or saliva using standard procedures. Exome capture was performed using an xGen target capture kit from IDT, and 99 base paired end sequencing on the Illumina platform (HiSeq 4000) was performed at the Yale 


\begin{tabular}{|c|c|c|c|c|c|}
\hline \multicolumn{3}{|c|}{ Clinical Features in Patient 1 and Others with CSCFS/FMD2 } & CSCFS & Patient 1 & FMD2 \\
\hline \multirow{2}{*}{\multicolumn{2}{|c|}{ Constitutional }} & Short stature & $\checkmark$ & $\checkmark$ & (Rare) \\
\hline & & Failure to thrive & $\checkmark$ & $\checkmark$ & \\
\hline \multirow{8}{*}{\multicolumn{2}{|c|}{ Face }} & Facial gestalt & $\checkmark$ & $\checkmark$ & \\
\hline & & Hypotonic face & $\checkmark$ & $\checkmark$ & \\
\hline & & Full cheeks & $\checkmark$ & $\checkmark$ & $\checkmark$ \\
\hline & & Macrostomia & $\checkmark$ & & \\
\hline & & Long philtrum & $\checkmark$ & $\checkmark$ & \\
\hline & & Prominent forehead/bitemporal narrowing ${ }^{a}$ & $\checkmark$ & $\checkmark$ & \\
\hline & & Anteverted nares & $\checkmark$ & $\checkmark$ & \\
\hline & & Broad nasal bridge & $\checkmark$ & $\checkmark$ & $\checkmark$ \\
\hline \multirow{4}{*}{\multicolumn{2}{|c|}{ Ears }} & Posteriorly rotated ears & $\checkmark$ & $\checkmark($ and small) & \\
\hline & & Recurrent otitis media & $\checkmark$ & $\checkmark$ & \\
\hline & & Conductive hearing loss, bilateral & $\checkmark$ & $\checkmark$ & \\
\hline & & Inner ear malformation & $\checkmark$ & & \\
\hline \multirow{5}{*}{\multicolumn{2}{|c|}{ Eyes }} & Strabismus & $\checkmark$ & $\checkmark$ & \\
\hline & & Dystopia canthorum/hypertelorism & $\checkmark$ & $\checkmark$ & \\
\hline & & Fullness of the upper and/or lower eyelid & $\checkmark$ & & $\checkmark$ \\
\hline & & Downslanted palpebral fissures & $\checkmark$ & $\checkmark$ & $\checkmark$ \\
\hline & & Ptosis & $\checkmark$ & $\checkmark$ & \\
\hline \multirow{3}{*}{\multicolumn{2}{|c|}{ Cardiovascular }} & Septal defect & $\checkmark$ & $\checkmark$ & \\
\hline & & Valve dysplasia & $\checkmark$ & $\checkmark$ & $\checkmark$ \\
\hline & & Aortic arch abnormalities & $\checkmark$ & & $\checkmark$ \\
\hline \multirow{4}{*}{\multicolumn{2}{|c|}{ Gastrointestinal }} & Feeding difficulties since birth & $\checkmark$ & $\checkmark$ & \\
\hline & & Gastroesophageal reflux & $\checkmark$ & & \\
\hline & & Gastrostomy tube & $\checkmark$ & & \\
\hline & & Gastrointestinal dysmotility & $\checkmark$ & & \\
\hline \multirow{2}{*}{\multicolumn{2}{|c|}{ Genitourinary }} & Small testis & $\checkmark$ & & \\
\hline & & Ectopic testis/cryptorchidism & $\checkmark$ & $\checkmark$ & $\checkmark$ \\
\hline \multirow[t]{13}{*}{ Skeletal } & General & Delayed bone age & $\checkmark$ & $\checkmark$ & \\
\hline & & Pectus excavatum & $\checkmark$ & (Mild) & $\checkmark$ \\
\hline & Spinal & Dorsal spine synostosis & $\checkmark$ & $\checkmark$ & \\
\hline & & Scoliosis & $\checkmark$ & (Mild) & $\checkmark$ \\
\hline & & Cervical vertebral fusion & $\checkmark$ & $\checkmark$ & $\checkmark$ \\
\hline & Limbs & Short extremities & $\checkmark$ & $\checkmark$ & \\
\hline & & Elbow contractures & & $\checkmark$ & $\checkmark$ \\
\hline & & Joint laxity & $\checkmark$ & $\checkmark$ & \\
\hline & Hands/feet & Brachydactyly & $\checkmark$ & $\checkmark$ & \\
\hline & & Cone-shaped epiphysis & $\checkmark$ & & \\
\hline & & Carpal/tarsal fusion & $\checkmark$ & $\checkmark$ & \\
\hline & & Pseudoepiphysis & $\checkmark$ & $\checkmark$ & \\
\hline & & Widely spaced first and second toes ${ }^{a}$ & & $\checkmark$ & $\checkmark$ \\
\hline \multirow{2}{*}{\multicolumn{2}{|c|}{ Integumentary }} & Soft/velvety skin & $\checkmark$ & $\checkmark$ & (Rare) \\
\hline & & Wrinkled skin of palms and soles & & $\checkmark$ & \\
\hline
\end{tabular}

(Yellow) Cardiospondylocarpofacial syndrome (CSCFS) features from Sousa et al. (2010), Le Goff et al. (2016), Morlino et al. (2018); (blue) frontometaphyseal dysplasia 2 (FMD2) features from Basart et al. (2015), Wade et al. (2016, 2017), Costantini et al. (2018); (green) features found in both CSCFS and FMD2; (red) novel phenotype.

a Not described, but pictured in said publications. 

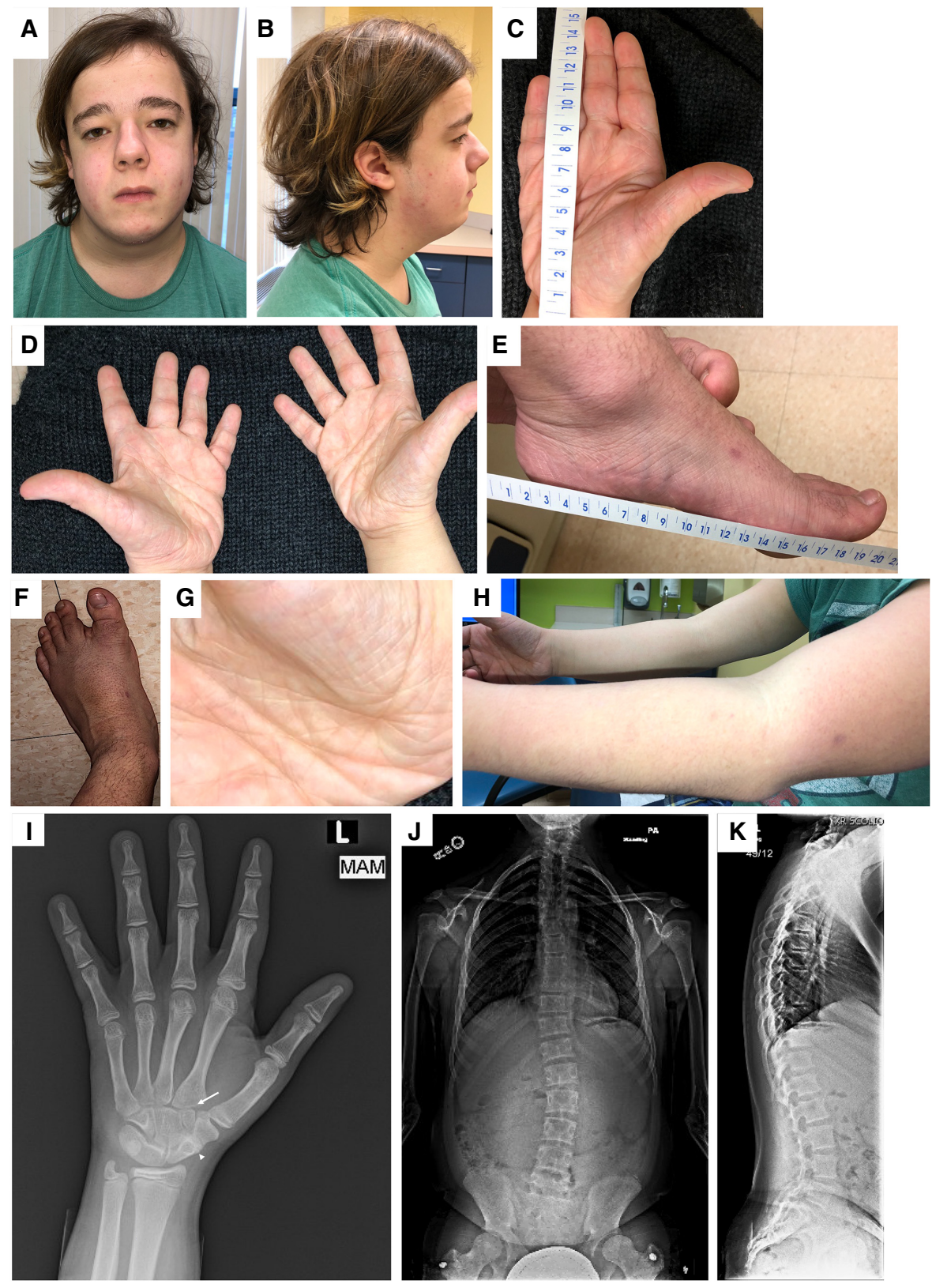

Figure 1. $(A, B)$ Portrait and profile of face. $(C, D)$ Small hands with brachydactyly. $(E, F)$ Small feet. Note widely spaced first and second toes as well as brachydactyly. $(G)$ Thin, wrinkled appearing skin of the palms. $(H)$ Maximum extension of the elbow. Note bilateral elbow contractures left more so than right with left maximally extended to $140^{\circ}$ and right to $150^{\circ}$. (I) Hand X ray demonstrating bilateral accessory proximal epiphyses within the second metacarpal (arrow) and synostosis between the right lunate and triquetrum (arrowhead). (J,K) Scoliosis survey demonstrating thoracolumbar scoliosis with minimal L5 on S1 anterolisthesis.

Center for Genome Analysis. The sequence reads were converted to FASTO format and were aligned to the reference human genome (hg19). GATK best practices were applied to identify genetic variants, and variants were annotated by ANNOVAR (Supplemental Table 1). 


\begin{tabular}{|c|c|c|c|c|c|c|c|}
\hline Gene & Genomic location & HGVS cDNA & $\begin{array}{l}\text { HGVS } \\
\text { protein }\end{array}$ & Zygosity & ClinVar ID & $\begin{array}{l}\text { Parent } \\
\text { of origin }\end{array}$ & $\begin{array}{c}\text { Variant } \\
\text { interpretation }\end{array}$ \\
\hline MAP3K7 & $\begin{array}{l}\text { Chr 6:91281524_91281526del } \\
\text { (GRCh37) } \\
\text { Chr 6:90571805_90571807del } \\
\text { (GRCh38) }\end{array}$ & $\begin{array}{l}\text { NM_145331.2: } \\
\text { c.125_127del }\end{array}$ & p.(Val42del) & Heterozygous & VCV000684731 & De novo & Likely pathogenic \\
\hline
\end{tabular}

\section{Variant Interpretation}

Comparison of Patient 1's clinical exome sequencing with his parents' research exome sequencing revealed a novel de novo variant in MAP3K7, c.125_127del, p.(Val42del) (Table 2). This variant was confirmed as present in the proband by Sanger sequencing. Although this variant has not been previously reported in the literature, it is closely situated to other known pathogenic variants, most closely to p.Arg44_Gly45del and p.Val50del (Le Goff et al. 2016). We classified p.(Val42del) as likely pathogenic as per recommended variant interpretation guidelines (Richards et al. 2015). It was determined to be a de novo variant (PS2) and absent from control individuals (PM2). Additionally, it causes a change in protein length (PM4) as p.(Val42del) lies in the protein kinase domain and, furthermore, it is the first amino acid in a nine-residue nucleotide binding region found near the amino terminus of the protein. Although the disease is likely too rare and with too much overlap with other disorders to say the patient's phenotype is highly specific for cardiospondylocarpofacial syndrome (CSCFS), his presentation certainly aligns with the diagnosis. Additionally, indel variants are recurrent in patients with CSCFS. Although this variant in MAP3K7 was deemed the most likely etiology of the patient's syndrome, two other genes were identified to have rare compound heterozygous variants (Supplemental Table 1). These genes (TCHH and EHHADH) were associated with phenotypes unrelated to the patient's findings and thus discarded as candidate genes. No rare hemizygous nor additional de novo variants were identified.

\section{SUMMARY}

The MAP3K7 gene is located on Chromosome 6q15 and encodes for the MAP3K7 protein, which is also known as TAK1 (transforming growth factor- $\beta$-activated kinase 1 ). TAK1 is a serine-threonine protein kinase that forms a complex with its associated binding proteins and then modulates a number of downstream effectors, including c-Jun amino-terminal kinases (JNKs), extracellular-signal regulated kinases (ERKs), p38 MAP kinase, and nuclear factor-kB, thereby affecting a wide range of cellular processes including cell growth and differentiation, immune function, stress responses, and apoptosis (Aashaq et al. 2019). The nearly ubiquitous expression of TAK1 and the early lethality of knockout mice speak to the importance of the protein in embryonic development (Sato et al. 2005; Ajibade et al. 2013).

MAP3K7 has been associated with human disease since 2016 with the simultaneous publication of reports of FMD2 and CSCFS (Le Goff et al. 2016; Wade et al. 2016). However, fewer than a dozen total MAP3K7 variants have been described to date in well-characterized patients. Many features of FMD2 and CSCFS overlap, with both syndromes featuring valvular heart disease, conductive hearing loss, certain facial features, genitourinary issues, scoliosis, and cervical vertebral fusion. Yet there are also key differences, with some CSCFS and FMD2 features that could be considered "opposites." CSCFS features long philtrum, brachydactyly, and joint laxity, whereas FMD2 features short philtrum, long slender digits, and joint 
contractures. There is some molecular evidence that differences between the two syndromes may be explained by variable effects on TAK1 function, as some FMD2 variants show increased TAK1 autophosphorylation, whereas some CSCF variants show decreased TAK1 autophosphorylation (Le Goff et al. 2016; Wade et al. 2016). These findings were noted in different experimental systems (overexpression vs. primary patient fibroblasts, respectively), however, and it will be interesting to see future experiments comparing CSCFS and FMD2 variants in the same context to see if TAK1 phosphorylation status can be used to assist with clinical diagnosis.

Although phosphorylation testing for Patient 1's variant was not performed, his phenotype falls most closely within the CSCFS spectrum, given his largely normal developmental status, short stature in the absence of severe scoliosis and osteosclerosis, and the characteristic features of carpal fusion, long philtrum, brachydactyly, and significant feeding issues from infancy into childhood. He has the novel feature of wrinkled skin of the hands and palms, which has not previously been described in CSCFS or FMD2, as well as velvety skin, which has been described in at least one other patient with CSCFS. However, the patient reported here is the first with CSCFS known to have flexion contractures of the elbow. He also has widely spaced first and second toes, which was observed in a patient with a deletion involving 6q15, where MAP3K7 resides (Klein et al. 2007). Although not explicitly commented on, medical photography demonstrates this finding in FMD2 as well (Wade et al. 2017). Still, the limited number of reports of patients with MAP3K7 variants makes it difficult to conclude whether these are true distinctions between CSCFS and FMD2.

Finally, although Crohn's disease, as seen in Patient 1, has complicated genetics, it is interesting that ulcerative colitis has been described as a rare manifestation of FMD2 (Basart et al. 2015). Additionally, the continued infections in Patient 1 could suggest some amount of immune dysregulation. MAP3K7 has roles in inflammation and the immune system (Ajibade et al. 2013); therefore, it is not clear if inflammatory bowel disease and recurrent otitis media are directly related to MAP3K7 variation or if these are significant but unrelated findings.

The novel MAP3K7 variant seen in Patient 1 is close to two previously recorded mutations associated with CSCFS, p.Arg44_Gly45del and p.Val50del, all within the kinase domain of the protein (Le Goff et al. 2016). All but one of the recorded variants in patients with FMD2 or CSCFS have been in the kinase domain (Morlino et al. 2018); however, it is important to note that a single missense variant in the carboxyl terminus of MAP3K7, p.Pro512Leu, is responsible for FMD2 in multiple unrelated individuals who typically have a more severe presentation than those with variants in the kinase domain (Wade et al. 2017). To date, all small in-frame deletions and splice variants (the latter of which results in an in-frame small duplication) have been associated with CSCFS instead of FMD2, whereas missense variants have caused both FMD2 and CSCFS (Le Goff et al. 2016; Wade et al. 2017). No frameshift or nonsense variants have been reported in this gene in association with CSCFS or FMD, suggesting that haploinsufficiency or loss of gene function are not causes of CSCFS or FMD2 phenotypes.

In summary, we present here a patient with a novel MAP3K7 variant, p.(Val42del), associated with CSCFS, including a new finding of wrinkled skin of hands and palms. Given that the two overlapping MAP3K7-associated syndromes have only recently been identified with a small number of patients, it remains unclear whether CSCFS and FMD2 are truly distinct syndromes or a spectrum of findings within a single disorder. At this time, with both overlap and consistent differences, as well as some genotype-phenotype relationships, it appears prudent to consider them distinct disorders on a clinical basis, with the expectation that additional patients and further molecular investigation will clarify the clustering of MAP3K7 phenotypes in the future. 
Competing Interest Statement

S.A.L. is part owner of Qiyas Higher Health, a start-up company unrelated to this work. No other authors have any disclosures to report.

Received January 23, 2020; accepted in revised form April 14, 2020

\section{ADDITIONAL INFORMATION}

\section{Database Deposition and Access}

The MAP3K7 variant described in this family was submitted to ClinVar (https://www.ncbi.nlm .nih.gov/clinvar), with accession number VCV000684731.1. Raw data were not deposited to public access databases in the absence of patient consent but may be available through correspondence with the authors.

\section{Ethics Statement}

This study was approved by the Yale University Institutional Review Board. The individuals referenced in this case report have provided written consent to participate in our research protocol and its publication of deidentified data and provided specific consent for the inclusion of medically relevant photography.

\section{Acknowledgments}

We thank the Yale New Haven Hospital for supporting the Pediatric Genomics Discovery Program, the Yale Center for Genome Analysis for performing whole-exome sequencing, and the Yale DNA Diagnostics Laboratory for confirming our novel variant. We also thank Sara and Jeffery Buell for their generous contributions to the Pediatric Genomics Discovery Program. We finally thank Patient 1 and his family for their willingness to share their stories with the medical community.

\section{Author Contributions}

F.A. analyzed data and wrote the manuscript. L.J. analyzed clinical data and contributed to writing the manuscript. W.J. analyzed sequencing data and critically reviewed the manuscript. J.M.M. analyzed clinical data and critically reviewed the manuscript. S.A.L. guided experimental design and data analysis and contributed to writing the manuscript.

\section{Funding}

This study was funded internally by the Yale New Haven Hospital.

\section{REFERENCES}

Aashaq S, Batool A, Andrabi KI. 2019. TAK1 mediates convergence of cellular signals for death and survival. Apoptosis 24: 3-20. doi:10.1007/s10495-018-1490-7

Ajibade AA, Wang HY, Wang RF. 2013. Cell type-specific function of TAK1 in innate immune signaling. Trends Immunol 34: 307-316. doi:10.1016/j.it.2013.03.007

Basart H, van de Kar A, Adès L, Cho TJ, Carter E, Maas SM, Wilson LC, van der Horst CM, Wade EM, Robertson $\mathrm{SP}$, et al. 2015. Frontometaphyseal dysplasia and keloid formation without FLNA mutations. Am J Med Genet A 167: 1215-1222. doi:10.1002/ajmg.a.37044

Costantini A, Wallgren-Pettersson C, Makitie O. 2018. Expansion of the clinical spectrum of frontometaphyseal dysplasia 2 caused by the recurrent mutation p.Pro485Leu in MAP3K7. Eur J Med Genet 61: 612-615. doi:10.1016/j.ejmg.2018.04.004

Klein OD, Cotter PD, Moore MW, Zanko A, Gilats M, Epstein CJ, Conte F, Rauen KA. 2007. Interstitial deletions of Chromosome 6q: genotype-phenotype correlation utilizing array CGH. Clin Genet 71: 260-266. doi:10 $.1111 /$ j.1399-0004.2007.00757.x

Le Goff C, Rogers C, Le Goff W, Pinto G, Bonnet D, Chrabieh M, Alibeu O, Nistchke P, Munnich A, Picard C, et al. 2016. Heterozygous mutations in MAP3K7, encoding TGF- $\beta$-activated kinase 1, cause cardiospondylocarpofacial syndrome. Am J Hum Genet 99: 407-413. doi:10.1016/j.ajhg.2016.06.005

Morlino S, Castori M, Dordoni C, Cinquina V, Santoro G, Grammatico P, Venturini M, Colombi M, Ritelli M. 2018. A novel MAP3K7 splice mutation causes cardiospondylocarpofacial syndrome with features of hereditary connective tissue disorder. Eur J Hum Genet 26: 582-586. doi:10.1038/s41431-017-0079-x 
COLD SPRING HARBOR

Molecular Case Studies
A novel MAP3K7 variant with mixed features

Richards S, Aziz N, Bale S, Bick D, Das S, Gastier-Foster J, Grody WW, Hegde M, Lyon E, Spector E, et al. 2015. Standards and guidelines for the interpretation of sequence variants: a joint consensus recommendation of the American College of Medical Genetics and Genomics and the Association for Molecular Pathology. Genet Med 17: 405-424. doi:10.1038/gim.2015.30

Sato S, Sanjo H, Takeda K, Ninomiya-Tsuji J, Yamamoto M, Kawai T, Matsumoto K, Takeuchi O, Akira S. 2005. Essential function for the kinase TAK1 in innate and adaptive immune responses. Nat Immunol 6: 10871095. doi:10.1038/ni1255

Sousa SB, Baujat G, Abadie V, Bonnet D, Sidi D, Munnich A, Krakow D, Cormier-Daire V. 2010. Postnatal growth retardation, facial dysmorphism, spondylocarpal synostosis, cardiac defect, and inner ear malformation (cardiospondylocarpofacial syndrome?)—a distinct syndrome? Am J Med Genet A 152A: 539546. doi:10.1002/ajmg.a.33277

Wade EM, Daniel PB, Jenkins ZA, Mclnerney-Leo A, Leo P, Morgan T, Addor MC, Adès LC, Bertola D, Bohring $A$, et al. 2016. Mutations in MAP3K7 that alter the activity of the TAK1 signaling complex cause frontometaphyseal dysplasia. Am J Hum Genet 99: 392-406. doi:10.1016/j.ajhg.2016.05.024

Wade EM, Jenkins ZA, Daniel PB, Morgan T, Addor MC, Adès LC, Bertola D, Bohring A, Carter E, Cho TJ, et al. 2017. Autosomal dominant frontometaphyseal dysplasia: delineation of the clinical phenotype. Am J Med Genet A 173: 1739-1746. doi:10.1002/ajmg.a.38267 


\section{COLD SPRING HARBOR Molecular Case Studies}

\section{A novel variant in MAP3K7 associated with an expanded cardiospondylocarpofacial syndrome phenotype}

Fatima AbuBakr, Lauren Jeffries, Weizhen Ji, et al.

Cold Spring Harb Mol Case Stud 2020, 6: a005207 originally published online April 16, 2020 Access the most recent version at doi: $10.1101 / \mathrm{mcs} .0005207$
Supplementary http://molecularcasestudies.cshlp.org/content/suppl/2020/04/17/mcs.a005207.D Material C1
License This article is distributed under the terms of the Creative Commons Attribution-NonCommercial License, which permits reuse and redistribution, except for commercial purposes, provided that the original author and source are credited.
Email Alerting Receive free email alerts when new articles cite this article - sign up in the box at the Service top right corner of the article or click here.

